\title{
Rendimiento físico de atletas varones de élite de baloncesto en silla de ruedas de la región del Biobío-Chile en inicio del período preparatorio: estudio transversal Physical performance of wheelchair basketball athletes with Paralympic projection of the Biobío-Chile region: a transversal study
}

*Cristián Luarte-Rocha, **Luis Felipe Castelli Correia de Campos, *Kevin Campos Campos, *Karina Alarcón Segura, ***Matías Henriquez, *Yazmina Pleticosic Ramírez,

****Mónica Fernández Muñoz, *****Raul Smith, ******Fernando Muñoz, *******Edison Duarte

*Universidad San Sebastián (Chile), **Universidad del Bío-Bío (Chile), ***Instituto Nacional de Rehabilitación Pedro Aguirre Cerda

(Chile), ****Universidad Adventista de Chile (Chile), *****Unidad de Ejercicio y Deporte Adaptado (Chile), ******Universidad Metropolitana de Ciencias de la Educación (Chile), *******Universidad Estatal de Campinas (Brasil)

Resumen. El presente estudio tuvo como objetivos caracterizar, comparar y relacionar las variables de rendimiento físico según clase deportiva de los para deportistas de elite de baloncesto en silla de ruedas de la región del Biobío-Chile, en el inicio del período preparatorio. La muestra estuvo formada por 22 para-deportistas varones de baloncesto en silla de ruedas (BSR) con discapacidad física que participan en torneos a nivel nacional. Los atletas fueron agrupados según la clasificación funcional: puntos altos (PA) (3.0 hasta 4.5) y puntos bajos (PB) (1.0 hasta 2.5). Se evaluó la potencia muscular por medio del Lanzamiento de Balón Medicinal (LBM), la capacidad de aceleración/propulsión y velocidad máxima en la silla de ruedas por medio de la prueba deVelocidad $20 \mathrm{~m}(\mathrm{~T} 20 \mathrm{~m})$, el manejo de la silla de rueda sin y con control de balón por medio de las Pruebas Eight y Eight-ball y la potencia aeróbica con la prueba continuo e incremental Octagon Multistage Fitness Test (OMFT). No se observaron diferencias significativas para las variables de rendimiento motor entre los grupos PB y PA $(p<.05)$. Por otro lado, el LBM presentó relaciones significativas con T20m $(\mathrm{r}=-.66, \mathrm{p}=.001)$ y la prueba Eight $(\mathrm{r}=.55, \mathrm{p}=.015)$, el T20m con la prueba Eight $(\mathrm{r}=-.73$, $\mathrm{p}=.000)$ y, el OMFT con las pruebas Eight $(\mathrm{r}=.58, \mathrm{p}=.018)$ y Eight-ball $(\mathrm{r}=.73, \mathrm{p}=.001)$. En conclusión, la clase deportiva, que se asocia a la funcionalidad muscular de los atletas, no presentó diferencias con el desempeño de los atletas investigados.

Palabras Clave: Rendimiento físico; Deporte Paralímpico; Baloncesto en Silla de Ruedas.

Abstract. The present study aimed to characterize, compare, and correlate the physical performance variables according to sport class of elite wheelchair basketball athletes from the Biobío-Chile region at the beginning of the preparatory period. Twenty-two male athletes from BSR with physical disabilities participated in national tournaments. The athletes were grouped according to sport class: high points - PA (3.0 to 4.5) and low points - $\mathrm{PB}$ (1.0 to 2.5). Muscle power was evaluated by means of the Medicine BallThrow (LBM), the acceleration / propulsion capacity and maximum speed in the wheelchair by means of the $20 \mathrm{~m}$ Speed test (T20m), the handling of the wheelchair without and with ball control through the Eight and Eight-ballTests and aerobic power with the continuous and incremental Octagon Multistage Fitness Test (OMFT). No statistical difference was observed for the motor performance variables between the PB and PA groups ( $\mathrm{p}<.05)$. On the other hand, the LBM presented significant relationships withT20m $(r=-.66, \mathrm{p}=.001)$ and the Eight test $(\mathrm{r}=.55, \mathrm{p}=.015)$, theT20m with the Eight test $(r=-.73, p=.000)$ and, the OMFT with the Eight $(r=.58, p=.018)$ and Eight-ball $(r=.73, p=.001)$ tests. In conclusion, the sports class, which is associated with the muscular functionality of the athletes, did not show differences with the performance of the investigated athletes.

Keywords: Athletes with disabilities; Physical performance; Paralympic Sport;Wheelchair Basketball.

\section{Introducción}

El baloncesto en silla de ruedas (BSR) es una de las principales modalidades paralímpicas para personas con discapacidad física (Solera et al., 2021, Sporner, 2009, Cavadon, 2015, Granados, 2015, Yanci, 2015, Oliveira, 2017), con su origen posterior a la segunda guerra mundial, en Inglaterra, en el hospital de Stoke Mandeville, en el año 1944 (de Mello, 2012, IPC, 2020).

La modalidad es regulada por la International Wheelchair Basketball Federation (IWBF) que establece

Fecha recepción: 22-10-21. Fecha de aceptación: 27-01-22

Cristián Luarte-Rocha

cristian.luarte@uss.cl las reglas oficiales de juego que son similares al convencional, presentando algunas adaptaciones, como el uso de la silla de ruedas, para facilitar la práctica para atletas con Discapacidad Física (DF) (IWBF, 2018; Marszalek, 2019). Además, la IWBF norma las especificaciones de equipos e instalaciones, coordinando los campeonatos mundiales de damas y varones, regulando también la clasificación deportiva de sus participantes (IPC, 2020). La clasificación funcional es un requisito obligatorio para la participación en competiciones oficiales, donde los atletas son agrupados en distintas clases deportivas $(\mathrm{CD})$ que legitima su participación.

La clasificación funcional en el BSR considera los movimientos funcionales para la práctica deportiva que incluyen la estabilidad del tronco, equilibrio sentado y el movimiento del tronco en los planos horizontal, fron- 
tal y sagital, como empujar y manipular la silla de ruedas, regatear y pasar, disparar y rebotar el balón, así como las observaciones de los atletas en juego (Souza, 2020, Lira et al., 2010).

De acuerdo con la funcionalidad presentada, cada atleta será incorporado en una clase deportiva (CD) que varía de 1.0 a 4.5 , considerando que, cuanto menor es su CD mayor es el compromiso motor que presenta el atleta (IWBF, 2014; Cavedón, 2018; Oliveira, 2017). Según Marzsalek et al. (2019), la importancia de la clasificación de los atletas, así como el tiempo de juego, el nivel del torneo o el resultado de juego, son útiles para que entrenadores y atletas realicen prácticas de entrenamiento adaptadas al esfuerzo del juego, logrando así, que los deportistas alcancen el nivel más alto según su $\mathrm{CD}$.

El BSR es un deporte intermitente que exige de esos atletas, niveles adecuados de fuerza en sus distintas manifestaciones; de velocidad y agilidad, resistencia aeróbica y anaeróbica para realizar acciones de alta intensidad con breves intervalos de tiempo para recuperación (Seron et al., 2019, Iturricastillo et al., 2016, Gil et al., 2015). Sumado a eso, los atletas necesitan óptimos niveles técnicos como el control del balón y manejo de la silla de ruedas para las acciones determinantes en la modalidad, tales como; lanzamientos, aceleraciones, desaceleraciones y cambios de dirección (Soylu, 2020, Molik, 2010; Croft, 2010;Weeisland, 2015; Molik, 2017).

En ese sentido, se considera fundamental el control y monitoreo de las diferentes variables asociadas al componente físico como elemento fundamental para que, el atleta pueda realizar las acciones técnico-tácticas de forma más eficiente y con mayor grado de resistencia a la fatiga (Campos et al., 2019).

Diversos estudios buscaron caracterizar la demanda impuesta a los atletas de BSR durante la competencia y el perfil de los atletas de BSR, de diferentes niveles competitivos. Respecto del primero punto, Sporner et al. (2009) identificaron que los atletas de élite de BSR recorren promedio de $2679,5 \pm 1103,6 \mathrm{~m}$ con intensidad promedio de $1,48 \pm 0,13 \mathrm{~m} / \mathrm{seg}$ y realizan cerca de 239,8 $\pm 60,6$ acciones de aceleración y desaceleración durante los partidos. Sumado a esos aspectos, Seron et al. (2019) identificaron, en su revisión sistemática, que los juegos oficiales de BSR, presentan alta demanda en relación con los componentes cardiovasculares, donde los atletas generalmente pasan aproximadamente 65\% del tiempo de juego en niveles intensos de frecuencia cardíaca y sobre $70 \%$ del $\mathrm{VO}_{\text {peak }}$.
En relación con la caracterización de la performance de los atletas según las clases deportivas, se puede mencionar estudios como el de Molik et al. (2010) que identificaron diferencias en el desempeño anaeróbico entre atletas puntos altos (3.0-4.5) de élite de BSR cuando comparados a los atletas puntos bajos (1.0-2.5). Del mismo modo, Cavadon et al. (2015) identificaron que atletas de puntos bajos presentaron menor desempeño en una batería de pruebas motoras específicas para el BSR comparado con los atletas con clasificación 3.0 o superior que, a su vez no presentaron valores superiores que los atletas de clase 1.0-1.5 y grupos de atletas 2.0-2.5, lo que implica que la $\mathrm{CD}$ de los atletas no fue determinante en el desempeño de pruebas específicas de esos atletas italianos de BSR. Más recientemente, Soylu et al. (2020) concluyeron que el rendimiento atlético en deportistas de BSR presenta relación con la clasificación deportiva. Los autores identificaron diferencias en variables de la fuerza muscular de las extremidades superiores, la capacidad aeróbica y anaeróbica entre atletas turcos de élite de BSR puntos altos (3.0$4.5)$ cuando fueron comparados a los atletas puntos bajos (1.0-2.5).

Actualmente en Chile, no se identifican investigaciones que hayan buscado caracterizar el perfil de atletas de élite de BSR y la comparación del desempeño según la clase deportiva de los atletas.

Así, el presente estudio tuvo como objetivos (i) evaluar las variables de rendimiento físico de potencia muscular, resistencia de la fuerza, velocidad, agilidad y la capacidad cardiorrespiratoria en el inicio del período preparatorio y, (ii) relacionar el desempeño físico según clase deportiva de los atletas de élite en la modalidad de baloncesto en silla de ruedas de la región del Biobío-Chile.

\section{Material y Métodos}

\section{Muestra}

En este estudio participaron 22 atletas varones de BSR con discapacidad física que participaron en torneos a nivel nacional. Las características de los atletas se describen en la tabla 1. Para el desarrollo del estudio los atletas fueron agrupados según la CD (puntos altos y puntos bajos) de acuerdo con lo propuesto por Molik et al. (2010). Se consideró 1.0-2.5 (puntos bajos) y de 3.04.5 (puntos altos). Todos los participantes fueron informados acerca de los objetivos del estudio, las implicancias de su participación y se explicó acerca de la posibilidad de retirarse en cualquier momento de la evaluación. 
Fueron solicitados para firmar el consentimiento informado de acuerdo con los principios de la declaración de Helsinki. El estudio fue aprobado por el comité de ética de la Universidad Diego Portales, n.001/2021.

Como criterio de inclusión, los atletas debían presentar más de un año de participación en la modalidad, con un entrenamiento regular de al menos dos veces a la semana y participación en todas las pruebas realizadas en el presente estudio. Como criterio de exclusión, los atletas no podrían presentar alguna lesión o cualquier otra condición patológica que interfiriese en los resultados y en la realización de todas las pruebas.

\section{Rendimiento Físico}

\section{Lanzamiento de Balón Medicinal (LBM)}

Esta prueba consistió en que el atleta lanzara el balón medicinal de $2 \mathrm{~kg}$ a la mayor distancia posible. Los atletas realizaron la prueba en su silla de ruedas específica de entrenamiento. El punto cero de la prueba es demarcado a partir de la extensión de los codos con los brazos frente al cuerpo y a la altura de los hombros. Para la demarcación de la distancia se utilizó una cinta métrica con precisión en centímetros (Stanley FatMax ${ }^{\circledR}$ 10/33“) fijada al suelo. Para el test se utilizó dos evaluadores, uno responsable por validar el lanzamiento y un segundo para registrar la distancia obtenida por el balón y su primer contacto con el suelo. Cada atleta realizó tres intentos partiendo con el balón en contacto con la región pectoral, altura esternal, con pausa de un minuto entre los intentos, registrando la mejor de ellas. La prueba presenta $r=.99$ para evaluar la potencia de miembros superiores (Stockbrugger \& Haennel, 2001).

\section{Test deVelocidad 20m (T20m)}

Esta prueba consiste en medir la velocidad máxima de los atletas por medio del tiempo en que el atleta recorre la distancia de 20 metros (Vanlandewijck, 1999). El protocolo utilizado fue similar a lo propuesto por Fierro, Villacieros y Perez-Tejero (2016), donde cada atleta tomó una posición en la línea de salida con las ruedas delanteras de la silla de ruedas en la línea demarcada como punto cero y orientados a permanecer con el tronco detrás de ella. En ese sentido, fueron ingresados dos postes en cada extremo de la línea para asegurarse de que el pecho del jugador o la rueda no cruzaran la línea antes del inicio de la prueba. La señal de inicio fue verbal y utilizó las palabras «listo» y luego «cuando quieras» para que los atletas tuvieran la libertad de realizar movimientos preparatorios de conduc- ción con el tronco y comenzar a impulsar la silla hacia adelante cuando estuvieran preparados. Dos evaluadores participaron del teste, el primero con el objetivo de validar la salida y el segundo posicionado al final del recorrido $(20 \mathrm{~m})$ para registrar el valor, en tiempo (segundos) con el uso de un cronometro digital (Vollo, VL$\left.1809^{\circledR}\right)$. Todos los atletas realizaron dos intentos válidos con pausa de 3 minutos entre los intentos. Se registró el menor tiempo realizado por el atleta, en segundos (Vanlandewijck, 1999).

\section{Prueba de agilidad Eight (T8) y Eight-ball (T8-ball)}

De acuerdo con Vanlandewijck, Daly, \& Theisen (1999), la prueba consiste en que, el atleta mueve su silla de ruedas alrededor de dos conos formando una figura de ocho durante un minuto. Para la preparación fueron utilizados 3 conos con distancia de cinco metros uno del otro en una disposición linear. Para la realización del teste los atletas fueron orientados a permanecer en una posición en la línea de salida con las ruedas delanteras de la silla de ruedas en la línea demarcada como punto cero, permanecer con el tronco detrás de ella, con y sin la posesión del balón. La señal de inicio fue verbal y utilizó las palabras «listo» y luego «cuando quieras»y, entonces los atletas iniciaban la prueba que consistió en el atleta realizar los intentos ultrapasando los conos formando un ocho, durante 1 minuto. En caso de los intentos con el balón, los atletas deberían botear el balón a cada 2 propulsiones en la silla de ruedas (de acuerdo con la regla del deporte). El tiempo fue controlado por un cronometro digital (Vollo, VL-1809 ${ }^{\circledR}$ ) y el puntaje consistió en la cantidad de veces en que el atleta pudo pasar completamente por el cono de inicio en cada intento (sin y con balón). Según Vanlandewijck et al. (1999) las pruebas presentan $r=.96$.

\section{Octagon Multistage Fitness Test (OMFT)}

Para determinar la potencia aeróbica de los atletas se utilizó el OMFT (Vanderthomenn et al., 2002). El OMFT es un test de intensidad progresivamente creciente, basado en el protocolo incremental descrito por Leger \& Boucher, (1980). A los sujetos se les solicita que giren alrededor de un curso octogonal delimitado por conos. Se requiere de una superficie mínima de 15 $\mathrm{m} \times 15 \mathrm{~m}$. Los cuatro lados principales del octágono tienen $11 \mathrm{~m}$ de largo y los cuatro lados cortos (ángulos), $2.83 \mathrm{~m}$ de largo. Las cuatro zonas de giros, a través de las cuales pasan los sujetos, se definen por dos conos «internos» que delimitan el ángulo del octógono y un cono «externo» colocado en la esquina de un cuadrado 
de $15 \mathrm{~m} \times 15 \mathrm{~m}$. La velocidad que determina la intensidad de la OMFT, se incrementa en etapas de un minuto de duración, con el uso de la retroalimentación auditiva (señal de «sonoro») a partir de una cinta de audio. En cada señal sonora, el sujeto tiene que estar dentro de la zona de giro. Esto da como resultado una velocidad inicial de rodadura de $6 \mathrm{~km} / \mathrm{h}$ e incrementos de $0.37 \mathrm{~km} / \mathrm{h}$. El test presentó $\mathrm{r}^{2}=.59$ para el VO2 $2_{\text {máx }}$ en atletas usuarios de silla de ruedas con diferentes deficiencias físicas. La ecuación utilizada para estimar el $\mathrm{VO} 2_{\text {máx }}$ de los atletas en la presente investigación y propuesta por Vanderthomenn et al. (2002) fue: VO2 ${ }_{\text {máx }}=$ $18.03+0.78 * \mathrm{NV}(\mathrm{NV}=$ número de vueltas $)$.

\section{Procedimientos}

Para el desarrollo del presente estudio, todos los participantes utilizaron sus sillas de ruedas deportivas y todos los equipamientos (ej. guantes, cinturón) que utilizan comúnmente en los partidos oficiales.

La semana anterior a la realización de los tests, todos atletas fueron invitados al local del entrenamiento e informados respecto a los procedimientos de recolección de datos. Todos realizaron la familiarización de las pruebas motoras para el desarrollo de este estudio con el objetivo de optimizar el tiempo en el día de las evaluaciones y aclarar dudas que eventualmente podrían surgir. Los participantes fueron instruidos a no ejecutar ningún tipo de actividad física moderada o vigorosa durante la semana previo a la evaluación. Cabe destacar que los atletas entrenan regularmente 3 veces a la semana con una duración de 60 minutos. Generalmente los entrenamientos están compuestos por sesiones específicas de estímulos técnico-tácticos.

La evaluación se realizó en inicio de la etapa preparatoria, donde los atletas ya presentaban 3 semanas de transición (etapa caracterizada con niveles bajos respecto al volumen e intensidad de los entrenamientos), considerando evaluación diagnóstica para la individualizaron de los entrenamientos según la posición de juego y funcionalidad.

Las mediciones se efectuaron en pista oficial de BSR, de madera flotante. Los testes motores fueron realizados en el mismo día, en un orden de realización partiendo de los testes más cortos e intensos seguido de los testes de mayor duración, con el fin de no ver afectado el rendimiento físico por la presencia de fatiga. Los testes fueron conducidos en 2 períodos (mañana y tarde). En la mañana se realizaron en secuencia los testes LBM y T20m. Para el período de la tarde, se realizó los testes de Agilidad Eight-ball y OMFT.
Previo a las mediciones, se realizó un calentamiento estandarizado de 15 minutos que consistió en ejercicios de movilidad articular incluidos al inicio e intermedio, entre los estímulos de propulsiones en sillas de ruedas en diferentes direcciones con ritmos variados y ejercicios de baja intensidad con el manejo de balón. La intención fue activar la musculatura y acercarse a los movimientos técnicos asociados a las pruebas que fueron sometidos sin producción de fatiga que inviabilizara la toma de datos.

\section{Análisis estadístico}

Todos los resultados fueron presentados en promedio y desviación estándar. La normalidad de los datos y la igualdad de las varianzas se evaluaron mediante las pruebas de Shapiro-Wilk y Levene.

Se utilizó la prueba $\mathrm{T}$ student para muestras independientes para determinar la diferencia entre variables de caracterización y del rendimiento físico de los atletas PB y PA. Se calculó el tamaño de efecto entre las variables de rendimiento físico por el procedimiento de cohen y la interpretación de los valores fue de $>.80$ : alto, $>.50$ y $<0.79$ : moderado, $>.25$ y $<.49$ pequeño, $\mathrm{y}<.25$ : trivial (Cohen, 1988).

La prueba de correlación de Pearson fue utilizada para relacionar las variables del rendimiento físico y Spearman entre la variable independiente CD y rendimiento físico. Se utilizó la escala de magnitudes propuesta por Hopkins et al. (2009): <.09, trivial; .10 .29 , pequeño; $<.30-.49$, moderado; $<.50-.69$, fuerte; $<.70-.89$, muy fuerte y $<.90-1.0$, casi perfecto, para determinar la relación entre las variables.

Los datos fueron tabulados a través del Software Microsoft Excel 2007® y analizados a través del software GraphPad Prism versión 9.0 para MACOs ${ }^{\circledR}$. El valor de significancia adoptado fue de $p \mathrm{~d} » .05$.

\section{Resultados}

La caracterización de los participantes es presentada en la tabla 1. No fueron identificados diferencias entre los grupos $\mathrm{PA}(\mathrm{n}=6)$ y $\mathrm{PB}(\mathrm{n}=16)$ para las variables de edad, tiempo de lesión (TL), talla y MC.

En relación con las variables de rendimiento físico, no fueron observadas diferencias significativas entre los atletas PA y PB $(p<.05)$. Sin embargo, el grupo PA presentó tamaño de efecto moderado (.68 y .62) para las pruebas T8-ball y del $\mathrm{VO}_{2}$ estimado en relación con el PB. Los resultados del rendimiento físico de los atletas de BSR son presentados en la figura 1. 
Tabla 1

Características de los atletas de baloncesto en silla de ruedas con proyección paralímpica de la región del Biobio-Chile

\begin{tabular}{|c|c|c|c|c|c|c|}
\hline tletas & $\mathrm{CD}$ & Tipo Discapacidad & Edad (años) & TL (años) & Talla (m) & $\mathrm{MC}(\mathrm{Kg})$ \\
\hline & 1 & Lesión Medular nivelT8 & 37 & 15 & 1,65 & 87 \\
\hline & 1 & artrogriposis & 24 & 24 & 1,62 & 64 \\
\hline & 1 & Lesión Medular nivelT8 & 54 & 18 & 1,7 & 67 \\
\hline & 1 & Lesión Medular nivel C6-C7 & 21 & 6 & 1,73 & 80 \\
\hline & 1 & Amputación MMII sobre rodilla & 26 & 26 & 1,24 & 79 \\
\hline & 1 & Lesión Medular nivelT8 & 37 & 15 & 1,65 & 87 \\
\hline & 1,5 & Mielomeningocele & 21 & 21 & 1,62 & 53 \\
\hline & 1,5 & Mielomeningocele & 27 & 27 & 1,6 & 95 \\
\hline & 2,5 & Mielitis transversa & 51 & 40 & 1,67 & 91 \\
\hline 0 & 2,5 & Artrosis bilateral de cadera & 37 & 4 & 1,65 & 76 \\
\hline 11 & 2,5 & Parálisis cerebral espástica & 44 & 44 & 1,62 & 65 \\
\hline 2 & 2,5 & Artrogriposis & 38 & 38 & 1,64 & 93 \\
\hline 3 & 2,5 & Mielomeningocele & 25 & 25 & 1,55 & 70 \\
\hline 4 & 2,5 & Lesión Medular nivel L2-L3 & 20 & 3 & 1,62 & 65 \\
\hline 15 & 2,5 & Parálisis Cerebral Espástica & 36 & 36 & 1,74 & 98 \\
\hline 6 & 2,5 & Mielitis transversa & 51 & 40 & 1,67 & 91 \\
\hline 7 & 4 & Hemiparesia espástica & 22 & 15 & 1,57 & 61 \\
\hline 10 & 4 & Amputación MMII sobre rodilla & 54 & 39 & 1,32 & 85 \\
\hline 9 & 4 & Hemiparesia espástica & 22 & 15 & 1,57 & 61 \\
\hline 0 & 4,5 & Amputación bajo rodilla derecha & 53 & 45 & 1,79 & 85 \\
\hline 1 & 4,5 & Amputación bajo rodilla izquierda & 35 & 1 & 1,76 & 74 \\
\hline 22 & 4,5 & Amputación bajo rodilla izquierda & 35 & 1 & 1,76 & 74 \\
\hline \multicolumn{3}{|c|}{ General $(n=22)(x \pm D E)$} & $35,3 \pm 11,9$ & $22,6 \pm 14,6$ & $1,62 \pm 0,13$ & $77,3 \pm 12,8$ \\
\hline \multirow{2}{*}{\multicolumn{3}{|c|}{$\begin{array}{l}\mathrm{PB}(\mathrm{n}=16)(\mathrm{x} \pm \mathrm{DE}) \\
\mathrm{PA}(\mathrm{n}=6)(\mathrm{x} \pm \mathrm{DE})\end{array}$}} & $34,3 \pm 11,4$ & $23,9 \pm 13,2$ & $1,63 \pm 0,11$ & $78,8 \pm 13,6$ \\
\hline & & & $36,8 \pm 14,2$ & $19,3 \pm 18,7$ & $1,63 \pm 0,18$ & $73,4 \pm 10,7$ \\
\hline
\end{tabular}
puntos altos. No se observaron diferencia entre los grupos PA y PB. ${ }^{*} p<.05$

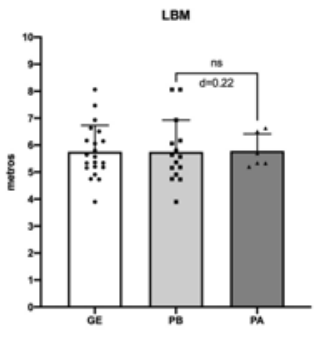

T8

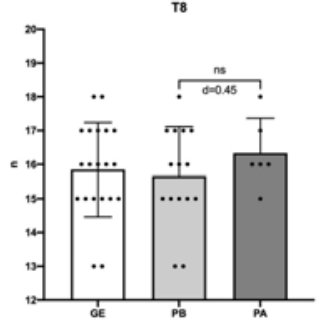

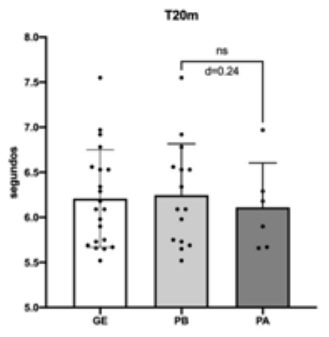

T8-ball
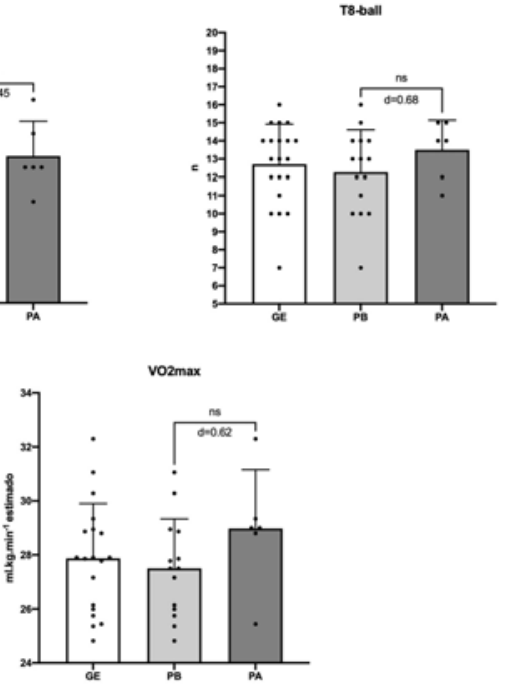

Figura 1. Rendimiento físico de los atletas PB y PA de baloncesto en silla de ruedas.

Nota: PA- Puntos Altos; PB - Puntos Bajos; LBM - Lanzamiento balón medicinal;T20m - Tiempo Sprint 20 metros; $\mathrm{n}$ - número de vueltas, T8 - prueba Eight;T8-ball - Eight-ball.

No fueron observadas relaciones significativas entre $\mathrm{CD}$ y variables del rendimiento físico para los atletas chilenos de BSR de proyección paralímpica. Por otro lado, fueron identificadas relaciones significativas inversamente proporcionales entre la prueba de potencia muscular LBM con la variable de sprint T20m $(\mathrm{r}=$ $.66, \mathrm{p}=.001)$ y proporcionalmente significativas con $\mathrm{T} 8$ $(\mathrm{r}=.55, \mathrm{p}=.015)$. Se observó relación significativa e inversamente proporcional entre capacidad de Sprint T20m con la pruebaT8 $(r=-.73$, «muy fuerte», $\mathrm{p}=.000)$. La capacidad aeróbica $-\mathrm{VO}_{2 \text { máx }}$ estimado fueron significativamente relacionadas con las pruebas T8 $(\mathrm{r}=.58$, «fuerte», $\mathrm{p}=.018)$ y $\mathrm{T} 8$-ball $(\mathrm{r}=.73$, «muy fuerte», $\mathrm{p}=.001)$. Los resultados son descritos en la tabla 2 .

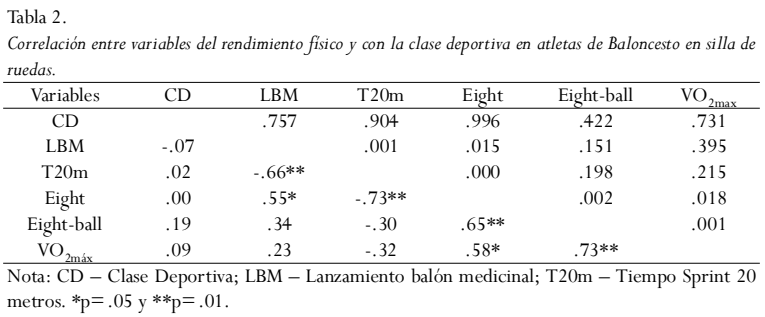

\section{Discusión}

Los principales resultados en esta investigación demostraron no haber diferencia estadística entre las pruebas de desempeño físico entre los atletas PA y PB. Molik et al. (2010) y Soylu et al. (2020) utilizaron la misma propuesta de división entre grupos y diferentemente de lo expuesto, observaron diferencias entre los grupos, lo que puede estar influenciado por el tipo de entrenamiento a que los atletas son sometidos, mientras que en ese estudio los atletas están en la etapa inicial de su período preparatorio general.

Diversos estudios presentan resultados significativamente diferentes en el rendimiento en pruebas físico-técnicas de atletas de BSR con menor y mayor compromiso motor (Vandanlewijck et al., 1999, Vanlandewijck et al., 2004, Molik et al., 2009, Cavadon, Zancanaro \& Milanese, 2018), considerando que el desempeño, es diferente, según las CD. En esta misma dirección, Gómez et al. (2015) evidenciaron que, si bien, se registran diferencias entre $\mathrm{CD}$ de puntos altos y puntos bajos, se identifican mayormente similitudes.

Por otro lado, también hay estudios en BSR que no identificaron diferencias significativas en todas las variables de desempeño y en todos los grupos de CD. Cavadon, Zancanaro \& Milanese (2015) y Gomes et al. (2015) identificaron diferencias significativas cuando comparados los atletas de CD 1.0 con atletas de CD 3.0 o superior. En nuestros hallazgos, podríamos mencionar que los atletas presentaron reducción de su performance durante el período de transición, lo que podría influenciar en mayor magnitud el potencial del entrenamiento de atletas que presentan menor compromiso motor, ya que solamente actividades de vida diaria no son intensas como exigidos durante el proceso de en- 
trenamiento, así como las diferencias discapacidades físicas que componen los grupos PA y PB.

La potencia muscular de los atletas se evaluó por medio del LBM. Prueba rápida, factible y accesible para medir el rendimiento en atletas paralímpicos (Gomes et al., 2021; Ribeiro et al., 2021). Para Ribeira Neto et al. (2021) la prueba de LBM es fiable y de fácil aplicación, que puede ser considerado para detección de talentos en la modalidad, así como una prueba control para progresión de los entrenamientos de atletas de BSR.

Los resultados en nuestro estudio no evidenciaron diferencia significativa entre PB y PA. Utilizando el mismo protocolo, Yanci et al. (2015) evaluaron 15 jugadores masculinos, divididos en dos grupos: puntos bajos y puntos altos. Se verificó resultados superiores para los atletas puntos altos $(5,16 \pm 0,40 \mathrm{~m}$ vs $4,54 \pm 0,86 \mathrm{~m})$ con

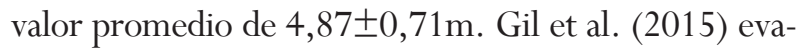
luaron 30 atletas varones españoles de 3 división por medio del test LBM. Se identificó un promedio de $3,78 \pm 0,66 \mathrm{~m}$. Cuando divididos en grupos según la CD $(<3.0$ y $>3.0)$ se identificó que el grupo de menor CD presentó resultados significativamente inferiores $(3,42 \pm 0,58$ vs 4,21 $\pm 0,52)$. Los autores sugieren que la biomecánica del momento de lanzamiento de jugadores con mayor musculatura de tronco y mayor estabilidad pélvica permitirá un lanzamiento con mayor distancia. Cabe mencionar que, en ese estudio se utilizó balón medicinal de 5kg y con lanzamiento por arriba de la cabeza, lo que imposibilita la comparación de los resultados. En ese mismo sentido, Souza (2013) identificó valores promedios de $8,7 \pm 1,6 \mathrm{~m}$ en la realización de la misma prueba en 19 atletas de BSR brasileños. Sin embargo, los resultados superiores reportados fueron influenciados por el uso del balón medicinal de $2 \mathrm{~kg}$.

Los atletas de BSR deben presentar óptima performance en su capacidad de aceleración y velocidad máxima, por considerarse como variables determinantes en la referida modalidad (Villacieros et al.,2020). La prueba de sprint en distancia de 20 metros está asociado a la velocidad de aceleración y máxima de atletas en silla de ruedas (Vandanlewijck et al., 1999) y puede ser considerado como una prueba control para monitorear el desempeño de los atletas de BSR (Loturco et al., 2020).

En nuestro estudio, no se observaron diferencias de los resultados promedios entre atletas PB y PA. Corroborando con nuestros hallazgos Gil et al. (2015) no observó diferencia entre atletas varones españoles de 3 división cuando divididos según la $\mathrm{CD}(<3.0: 5,62 \pm 0,45 \mathrm{~s}$ vs >3.0: 5,67 $\pm 0,48 \mathrm{~s})$. Yanci et al. (2015), evaluó a 15 atletas masculino de BSR de primera división de la liga española e identificó valores promedios de 5,16 $\pm 0,18 \mathrm{~s}$.

A diferencia de los autores anteriores (Gil et al., 2015; Yancy et al., 2015) García-Fresneda \& Carmona (2021) encontraron fuertes correlaciones entre los test de sprint de corta distancia $(3,5$ y $12 \mathrm{~m})$ con la CD en 16 atletas del sexo femenino del equipo de BSR de España. Las atletas fueron dividas en cuatro grupos: grupo I (clase 1 y 1.5), grupo II (clase 2 y 2.5), grupo III (clase 3 y 3.5) y grupo IV (clase 4 y 4.5), donde se observó resultados inferiores para el grupo I comparado al grupo IV $(\mathrm{p}<.05)$. Además, una fuerte asociación significativa entre la puntuación individual de la CD y los Sprint de distancia corta $(p<0.5)$. Por lo tanto, si bien, el entrenamiento y la experiencia técnica contribuyen en obtener mejores resultados en sprint de cortas distancias, la funcionalidad posee un rol más importante. En ese sentido, Villacieros et al. (2020) destacan la importancia e influencia de los niveles de fuerza de las musculaturas de rotación de hombro, flexión y extensión de codo, en miembros dominantes, para adquisición de mayores niveles de velocidad máxima en los atletas de BSR, así como en acciones como pasar, botear y frenar.

Loturco et al. (2020) evaluaron 11 atletas varones de élite brasileños con CD superior 3.0 y el valor pro-

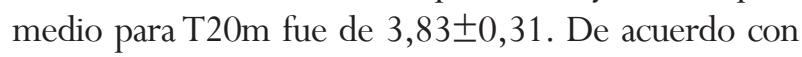
los autores, se puede observar diferencia de performance de los atletas de BSR, según el nivel competitivo de los atletas. A pesar de que, la muestra del presente estudio ha participado en competencias en nivel nacional, los resultados arrojan una menor performance en la velocidad con los resultados presentados en la literatura. Tales aspectos pueden estar asociados a diversos factores relacionados con la performance como la calidad de la silla, calibración de los neumáticos, la técnica de los atletas, nivel de entrenamiento y actividad muscular (Iturricastillo et al., 2018), lo que compromete la comparación ya que los estudios no presentan informaciones respecto a eses factores.

El metabolismo anaeróbico se asocia a las acciones determinantes en el BSR y que generalmente son estímulos de corta duración y alta intensidad, por otro lado, el metabolismo aeróbico es considerada la capacidad predominante en BSR, que permite no solamente una rápida recuperación de los estímulos de alta intensidad, como el atleta soportar el tiempo total de juego (Vanlandewijck et al., 2004). Una de las principales variables para mensurar la capacidad aeróbica en paraatletas es el consumo máximo de oxígeno $-\mathrm{VO}_{2 \text { máx }}$, que puede ser mensurado de forma directa o indirecta (Cam- 
pos et al., 2015).

Los resultados del presente estudio presentaron valores promedios similares a los identificados en investigaciones previas, tanto para atletas PA y PB. Entretanto, se destaca la dificultad de comparar los resultados debido a los diferentes protocolos utilizados en la literatura consultada (Granados, Iturricastillo, Lozano \& Yanci, 2016).

Conners et al (2020) en su estudio con jóvenes de BSR, realizaron un test de capacidad aeróbica en cicloergómetro con valores de $\mathrm{VO} 2_{\text {máx }}$ promedio de $22 \pm 6 \mathrm{ml} / \mathrm{kg}^{-1} / \mathrm{min}^{-1}$. A su vez, Pérez, Rabadan, Pacheco \& Sampedro (2002), establece una correlación positiva entre la distancia recorrida y elVO2 ${ }_{\text {maxx }}$. En este estudio, los atletas se distribuyeron en dos categorías: Categoría A (1.0 a 2.5 puntos) y Categoría B (3.0 a 4.5 puntos). Los resultados obtenidos presentaron promedio de $1562,6 \pm 315,8 \mathrm{~m}, \mathrm{VO} 2_{\text {máx }}$ absoluto $=2,9 \pm 0,4 \mathrm{l} / \mathrm{min} \mathrm{y}$ $\mathrm{VO} 2_{\text {máx }}$ relativo $=40,5 \pm 6,9 \mathrm{ml} / \mathrm{kg} / \mathrm{min}$, siendo la correlación de $\mathrm{r}=.85(p=.01)$ entre distancia recorrida $\mathrm{y}$ $\mathrm{VO} 2_{\text {max }}$

Iturricastillo, Yanci \& Granados (2015), midieron los parámetros de $\mathrm{VO} 2_{\text {máx }}$ por medio del protocolo Multistage fitness test (MSFT). Participaron 8 atletas españoles de un equipo de primera división con valores promedios de $30,2 \pm 4,5 \mathrm{ml} / \mathrm{kg} / \mathrm{min}$. Vanlandewijck et al. (1999) evaluaron 46 atletas belgas masculino de BSR, por medio de la prueba de 25 metros adaptado, versión original de Léger \& Lambert (1982) y protocolo en ergoespirometria, obteniendo valores promedios de $\mathrm{VO} 2_{\text {máx }}$ de 2208,3 $\pm 461,6 \mathrm{ml} / \mathrm{min}$.

La resistencia muscular, agilidad y el manejo de la silla de rueda son factores determinantes en el rendimiento deportivo en el BSR, donde los jugadores deben realizar repetidamente aceleraciones y desaceleraciones con cambios repentinos de dirección inúmeras veces durante el partido (Sugiyama et al., 2021). El test Eight y Eight ball, tiene la finalidad de evaluar esas características, asociadas también al control del balón, constituyen como aspectos relevantes a ser considerados durante el periodo de preparación de atletas de BSR (Vanlandewijck et al., 1999).

En el estudio del mismo autor mencionado, los resultados promedios fueron de 17,3 $\pm 1,2$ pasajes para la prueba T8. Souza (2013) obtuvo un promedio de 16,3 $\pm 1,7$ pasajes. Avello, Paredes \& Paredes (2018) evaluaron 7 atletas de elite de BSR chilenos y presentaron promedio de $14,5 \pm 1,9$ a $17,0 \pm 2,6$ pasajes entre los grupos experimental y control.

Los resultados de esta investigación, con relación al
T8 fueron inferiores a los obtenidos en los estudios que utilizaron el mismo protocolo, evidenciando que los atletas de esta pesquisa pudieron presentar menor habilidad de manejo en silla de ruedas y una menor resistencia muscular periférica de los miembros superiores (Weissland, Faupin, Borel, Berthoin \& Lepretre, 2015). Por otro lado, no fueron identificados estudios que utilizaron el test asociado al control del balón como aquí presentado, lo que podría representar una mayor especificidad con la actividad competitiva.

El estudio presenta debilidades en que la muestra reducida no se puede generalizar los resultados a los atletas de BSR chilenos, pero evidentemente la fortaleza principal del estudio es que, de acuerdo a literatura consultado, el estudio es pionero en presentar la característica y perfil de los atletas de elite en la modalidad durante el período preparatorio y sugiere la aplicación de una batería de testes capaz de evaluar las principales aspectos determinantes y predominantes en la modalidad.

\section{Conclusiones}

El estudio tuvo como objetivo evaluar las variables de rendimiento físico de atletas de BSR con proyección paralímpica de la región del Biobío-Chile. No se observó diferencia estadística entre los grupos ( $\mathrm{PB}$ y $\mathrm{PA}$ ) cuando fueron comparados. Tal aspecto es divergente de los resultados presentados en diversas investigaciones y puede estar condicionado al sistema de entrenamiento a los cuales los atletas son sometidos, una vez que la literatura demuestra la diferencia entre los grupos analizados y como principal justificación son cuestiones de mayor funcionalidad y menor comprometimiento muscular en atletas con $\mathrm{CD}$ mayores.

Agradecimientos

Se agradece de forma especial a los Atletas de proyección Paralímpica de la Región del Biobío, a la Universidad San Sebastián y al grupo de investigación GIDEPAUSS.

\section{Referencias}

Avello, J., Paredes, N. \& Paredes, G. (2018). Implementación de estrategias mixtas para mejorar la agilidad en atletas pertenecientes al equipo de básquetbol en silla de ruedas, Grand Bull de Concepción. (Tesis de Licenciatura en Educación). Uni- 
versidad San Sebastián, Concepción, Chile.

Castelli Correia de Campos, L. F., Ribeiro da Luz, L. M., Luarte Rocha, C. E., Diehl Nogueira, C., Labrador Roca, V. \& Irineu Gorla, J. (2019). Validation of Test Studies for the Analysis of Aerobic Power in Tetraplegic Athletes. Apunts. Educación Física y Deportes, 135, 68-81.

Cavedon, V., Zancanaro, C. \& Milanese, C. (2018). Anthropometry, Body Composition, and Performance in Sport-Specific Field Test in Female Wheelchair Basketball Players. Front. Physiol, 9(568)

Cavedon, V., Zancanaro, C. \& Milanese, C. (2015). Physique and performance of young wheelchair basketball players in relation with classification. PLoS One, 10(11).

Costa e Silva, A. A., Borges, M., Faria, F. R., Campos, L. F., Yamagute, P. C., Gatti, A.M. \& Gorla.J. I. (2014). Validation of battery skill tests to wheelchair handball athletes. Rev.int.med.cienc.act.fis.deporte, 17(65), pp. 167-182.

Cohen, J. (1988). Statistical power analysis for the behavioral sciences. Hillsdale, N.J.: L. Erlbaum Associates

Conners, R.T., Elliott, J.M., Kyle, D.L., Solomon, S., \& Whitehead, P.N. (2020). Physiological Responses of Youth Players During Wheelchair Basketball Games. EUJAPA, 13(2), 9.

Croft L, Dybrus S, Lenton J. \& Goosey-TolfreyV. (2010). A Comparison of the physiological demands of wheelchair basketball and wheelchair tennis. Int J Sports Phys Perform. 5:301-315.

Cumellas, M. \& Estrany, C. (2006). Discapacidades motoras y sensoriales en primarias. INDE.

De Mello, M. \& Winckler, C. (2012). Esporte Paralímpico. São Paulo: Editora Atheneu.

Souza, L. et al (2020). Limitaciones y Posibilidades en el Entrenamiento del Baloncesto en Silla de Ruedas. Rev.peru.cienc.act.fis.deporte ,7(4):1036 - 1044.

Fréz, A., Souza, A. \& Quartiero, C. (2015). Funtional performance of wheelchair basketball players whith spinal cord injury. Acta. Físiat. 22(3), pp. 141-144.

Frenz, W. (2015). Handbook of EU Competition. Springer.

García Fresneda, A. \& Carmona, G. (2021). Short Distance Sprint Performance in EliteWheelchair Basketball Women Players: Influence of Functional Classification. Apunts. Educación Física y Deportes, 144, 75-80.

Gil, S., Yanci, J., Otero, M., Olasagasti, J., Badiola, A., Bidaurrazaga-Letona, I., Iturricastillo, A. \& Granados,
C. (2015). La clasificación funcional y el rendimiento de las pruebas de campo en jugadores de baloncesto en silla de ruedas. Revista de cinética humana, 46(1) 219-230.

Gómez, A. M., Molik, B., Morgulec-Adamowicz, N. \& Szyman, J. R. (2015). Performance analysis of elite women's wheelchair basketball players according to team-strength, playing-time and players' classification. International Journal of Performance Analysis in Sport, 15(1), 268-283.

Gomes Costa, R., Dorneles, J., Lopes, G. H., Gorla, J. \& Ribeiro Neto, F. (2021). Medicine Ball Throw Responsiveness to Measure Wheelchair Basketball Mobility in Male Players, Journal of Sport Rehabilitation (published online ahead of print 2021). Retrieved Aug 22, 2021, from https:// journals.humankinetics.com/view/journals/jsr/ aop/article-10.1123-jsr.2020-0222/article-10.1123jsr.2020-0222.xml

Goosey-Tolfrey, V. (2010). Wheelchair sport: A complete guide for athletes, coaches, and teachers. Human Kinetics.

Gorgatti, M. \& Bohme. (2003). Autenticidade cientifica de um teste de agilidade para individuos em cadeira de rodas. Rev. Paul. Educ. Fis. Sao Paulo, 17(1), pp. 41-50.

Gorla, J., Costa e Silva, A., Costa, L. \& Campos, L.F. (2011). Validacao da batería «Beck» de testes de habilidades para atletas brasileiros de «rugby» em cadeira de rodas. Rev. Bras. Educ. Fís. Esporte Sao Paulo, 25(3), pp. 473-86.

Granados, C., Iturricastillo, A., Lozano, L. \& Yanci, J. (2016). Effects of intermittent high intensity training in wheelchair basketball players physical performance. Rev. Cultura, Ciencia y Deporte, 33(11), pp. 235-240.

Granados, C., Yanci, J., Badiola, A., Iturricastillo, A., Otero, M., Olasagasti, J., ... Gil, S. M. (2015). Anthropometry and Performance inWheelchair Basketball. Journal of Strength and Conditioning Research, 29(7), 1812-1820.

Hernández, R., Fernández, C. \& Baptista P. (2014). Metodología de la Investigación. ( $6^{\mathrm{a}}$ ed.) México: McGraw-Hill.

Hopkins, W.G., Marshall, S.W., Batterham, A.M. \& Hanin, J. (2009). Progressive statistics for studies in sports medicine and exercise science. Medicine and Science in Sports and Exercise, 41(1), 3-12.

IPC. (2020). Paralympic Sports: Wheelchair Basketball. https: / / www.paralympic.org/wheelchair-bas- 
ketball

International Wheelchair Basketball Federation. (2014). IWBF Official Player Classification Manual 2014. https: / /iwbf.org/wp-content/uploads/2018/09/ PLAYER-CLASSIFICATION-MANUAL-2014SPANISH.pdf

Iturricastillo, A., Yanci, J. \& Granados, C. (2015). Physical performance, physiological responses and perceived exertion effort in Multi-Stage fitness test in wheelchair basketball players. Revista internacional de deportes colectivos. 23, pp. 5-13.

Iturricastillo, A., Yanci, J., Barrenetxea, I. \& Granados, C. (2016). Análisis de la intensidad de juego durante los partidos de play-off en jugadores de baloncesto en silla de ruedas. Retos, 30, pp. 50-58.

Lira, C.A., Vancini, R.L., Minozzo, F.C., Sousa, B.S., Dubas, J.P., Andrade, M.S., Steinberg, L.L. \& da Silva,A.C. (2010). Relationship between aerobic and anaerobic parameters and functional classification in wheelchair basketball players. Scandinavian journal of medicine \& science in sports, 20(4), 638-643.

Léger, L. \& Lambert, J.A. (1982). Maximal multistage 20-m shuttle run test to predict VO2 max. Eur J Appl Physiol Occup Physiol, 49(1), pp. 1-12.

Léger, L. \& Boucher R. (1980). An indirect continuous running multistage field test: the Université de MontréalTrackTest. Canadian journal of applied sport sciences, 5(2), pp. 77-84.

Loturco, I., McGuigan, M.R., Reis, V.P., Santos, S., Yanci, J., Pereira, L.A. \&Winckler, C. (2020). Relationship Between Power Output and Speed-Related Performance in Brazilian Wheelchair Basketball Players. Adapted Physical Activity Quarterly, 37(4), 508517.

MarszaBek, J., Gryko, K., Kosmol, A., MorgulecAdamowicz, N., Mróz, A. \& Molik, B. (2019). Wheelchair Basketball Competition Heart Rate Profile According to Players' Functional Classification, Tournament Level, Game Type, Game Quarter and Playing Time. Frontiers in Psychology, 10.

Molik, B., Kosmol, A., Morgulec'Adamowicz, N., Laskin, J., Jezior, T. \& Patrzalek, M. (2009). Game efficiency of elite female wheelchair basketball players during world championships (Gold cup). European Journal of Adapted Physical Activity, 2(2), pp. 26-38.

Molik, B., Laskin, J.J., Kosmol, A., Skucas, K. \& Bida, U. (2010). Relationship Between Functional Classification Levels and Anaerobic Performance of
Wheelchair Basketball Athletes. Research Quarterly for Exercise and Sport, 81(1), 69-73.

Molik, B., Kosmol, A., Morgulec-Adamowicz, N., Lencse-Mucha, J., Mróz, A., Gryko, K. \& MarszaBek, J. (2017). Comparison of Aerobic Performance Testing Protocols in Elite MaleWheelchair BasketbaIl Players. Journal of Human Kinetics, 60(1), 243254.

Pérez, J., Rabadan, M., Pacheco, J. \& Sampedro, J. (2002). Evaluación de la potencia aeróbica máxima en jugadores de baloncesto en silla de ruedas de alto nivel mediante test de campo. https:// www.cienciadeporte.com/images/congresos/ madrid/Rendimiento\%20Deportivo / Entrenami en to\% 20 Deportivo/ Evalua $\mathrm{P} / \mathrm{C} 3 \% \mathrm{~B} 3 \mathrm{n} \% 20 \mathrm{de} / 20 \mathrm{k} \% 20$ potenci $\% 20 \mathrm{ar} / \mathrm{C} 3 \% / \mathrm{B} 3 \mathrm{bicaPDF}$.

Reverdito, R., Scaglia, A. \& Montaner, P. (2013). Pedagogía do Esporte:Aspectos conceituais da competição e estudos aplicados. São Paulo: Phorte.

Ribeiro Neto, F., Loturco, I., Henrique Lopes, G., Rodrigues Dorneles, J., Irineu Gorla, J., \& Gomes Costa, R. R. (2021). Correlations Between Medicine Ball Throw With Wheelchair Mobility and Isokinetic Tests in Basketball Para-Athletes. Journal of sport rehabilitation, 1-5.

Salkind, N. (1998). Métodos de Investigación. México: Pearson.

Santana, V. \& Duarte, E. (2009). Síndrome de Down: Informações, caminhos e histórias de amor. São Paulo: Phorte.

Sanz, D. \& Reina, R. (2014). Actividades físicas y Deportes Adaptados Para Personas con Discapacidad. España: Paidotribo.

SENADIS. (2010). Manual sobre Ley N²0.422: Establece normas sobre Igualdad de Oportunidades e Inclusión Social de Personas com Discapacidad. https: / / www.senadis.gob.cl/descarga/i/655

Seron, B., Oliveira de Carvalho, E., Greguol, M. (2019). Analysis of Physiological and Kinematic Demands of Wheelchair Basketball Games-A Review. Journal of Strength and Conditioning Research, 33(5), pp.1453-1462.

Souza, M. (2013). Desempenho motor em atletas practicantes de basquete en cadeira de rodas. (Trabalho de Conclusão para obtenção do título de Bacharel em Educação Física). Campinas, Brasil.

Soylu, Ç., Y1ld1r1m, N. Ü., Akalan, C., Ak1noðlu, B. \& Kocahan, T. (2020). The Relationship Between Athletic Performance and Physiological Characteristics in Wheelchair Basketball Athletes. 
Research Quarterly for Exercise and Sport, 1-12. Sporner, M. L., Grindle, G. G., Kelleher, A., Teodorski, E. E., Cooper, R. \& Cooper, R. A. (2009). Quantification of Activity During Wheelchair Basketball and Rugby at the National Veterans Wheelchair Games: A Pilot Study. Prosthetics and Orthotics International, 33(3), 210 217.

Stockbrugger, B. A. \& Haennel, R. (2001). Validity and reliability of a medicine ball explosive power test. Journal of Strength and Conditioning Research, 15(4), pp. 431-438.

Sugiyama, T., Maeo, S., Kurihara, T., Kanehisa H. \& Isaka, T. (2021). Change of Direction Speed Tests in Basketball Players: A Brief Review of Test Varieties and Recent Trends. Front. Sports Act. Living 3:645350.

Tachibana, K., Mutsuzaki, H., Shimizu,Y., Doi, T., Hotta, K. \& Wadano, Y. (2019). Influence of Functional Classification on Skill Tests in Elite Female Wheelchair Basketball Athletes. Medicina, 55(11), pp. 1-10.

Torralba, M., Braz, M. \& Rubio, M. (2014). La motivación en el deporte adaptado. Journal Psychology, Society \& Education, 6(1), pp. 27-40.

Tweedy, S. \& Vanlandewijck, Y. (2011). International Paralympic Committee position stand-backround and scientific principles of classification in Paralympic sport. Br J Sports Med, 45(4), pp. 259-69.

Vanderthommen, M., Francaux, M., Colinet, C., Lehance, C., Lhermerout, C., Crielsard, JM. \& Theisen, D. (2002). A multistage field test of wheelchair users for evaluation of fitness and prediction of peak oxygen consumption. Journal of rehabilitation Research and development, 39(6), pp.685-692.

Vanlandewijck, Y., Daly, D. \& Theisen, D. (1999). Field test evaluation of aerobic, anaerobic, and Wheelchair Basketball skill performances. Int Journal Sports Med. 20(8), pp. 548-554.

Vanlandewijck, Y., Evaggelinou, C., Daly, D., Verellen, J., Van Houtte, S. \& Aspeslagh, V. (2004). The relationship between functional potential and field performance in elite female wheelchair basketball players. J. Sports Sci. 22(7), pp. 668-675.

Weissland, T., Faupin, A., Borel, B., Berthoin, S. \& Lepretre, P. (2015). Effects of modified Multistage FieldTest on Performance and Physiological responses en wheelchair Basketball players. Rev. BioMed research international, 2015.
Yanci, J. Iturricstillo, A. Lozano, L. \& Granados, C. (2015). Physical fitness analysis according the functional classification in national wheelchair basketball players. Rev. Int. De ciencias del deporte, 11(40), pp. 173-185.

Zucchi, D. (2001). Deporte y discapacidad. Revista Digital Efdeportes, 7(43).

Villacieros, J., Pérez-Tejero, J., Garrido, G., Grams, L., López-Illescas, Á., \& Ferro, A. (2020). Relationship between Sprint Velocity and Peak Moment at Shoulder and Elbow in Elite Wheelchair Basketball Players. International journal of environmental research and public health, 17(19), 6989. https://doi.org/10.3390/ijerph17196989

Ferro, A.,Villacieros, J., \& Pérez-Tejero, J. (2016). Sprint Performance of Elite Wheelchair Basketball Players: Applicability of a Laser System for Describing the Velocity Curve, Adapted Physical Activity Quarterly, 33(4), 358-373. https:// journals.humankinetics.com/view/journals/apaq/ 33/4/article-p358.xml

Iturricastillo Urteaga, A., Yanci Irigoyen, J., Barrenetxea Iriondo, I., \& Granados Dominguez, C. (2016). Análisis de la intensidad de juego durante los partidos de play-off en jugadores de baloncesto en silla de ruedas (Game intensity analysis of wheelchair basketball players during play-off matches). Retos, 30, 54-58. https://doi.org/10.47197/ retos.v0i30.39660

Solera, B., Flores, P., Anversa, A. L., Caraçato, Y. M., de Souza, V. de F., \& Herold athletes perceptions about social inclusion through sport). Retos, 42, 655661. https://doi.org/10.47197/retos.v42i0.85713

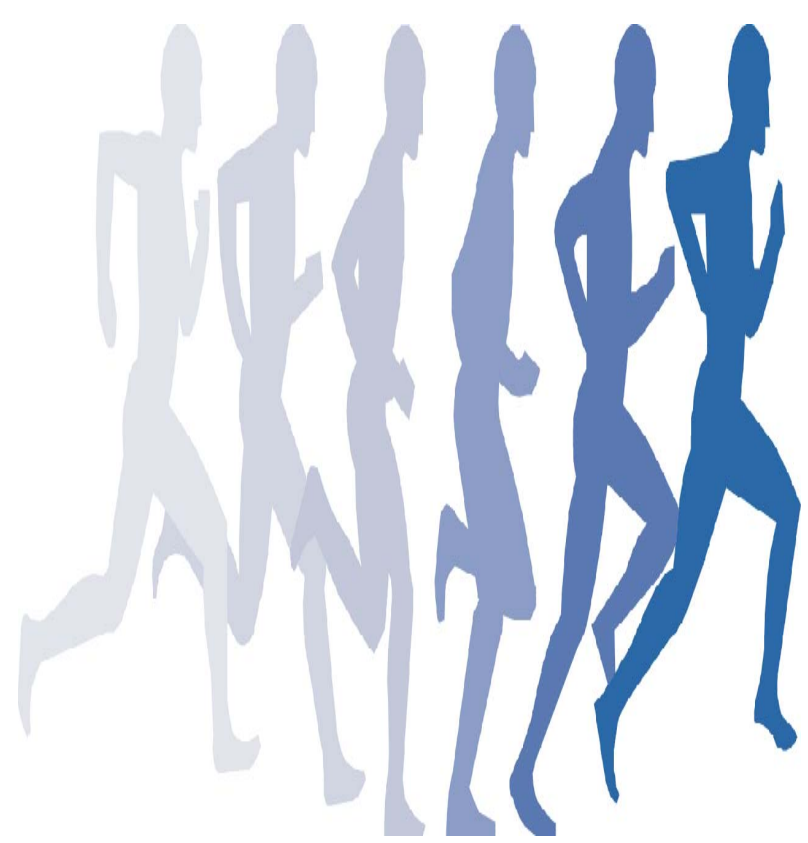

